\title{
A wind-tunnel study of the wake development behind wind turbines over sinusoidal hills
}

\author{
Ann Hyvärinen ${ }^{1}$, Giovanni Lacagnina ${ }^{1,2}$ and Antonio Segalini ${ }^{1,3}$ \\ ${ }^{1}$ Linné Flow Centre and STandUP for Wind, KTH Mechanics, Royal Institute of Technology, SE-100 44 Stockholm, Sweden \\ ${ }^{2}$ Faculty of Engineering and the Environment, University of Southampton, Southampton SO167QF, UK \\ ${ }^{3}$ Queen Mary University of London, Mile End Road, London E1 4NS, UK
}

\begin{abstract}
In the present work, the wake development behind small-scale wind turbines are studied when introducing local topography variations consisting of a series of sinusoidal hills. Additionally, wind-tunnel tests with homogeneous and sheared turbulent inflows were performed to understand how shear and ambient turbulence influence the results. The scale of the wind-turbine models was about 1000 times smaller than full-size turbines, suggesting that the present results should only be qualitatively extrapolated to real-field scenarios. Wind-tunnel measurements were made by means of stereoscopic particle image velocimetry to characterise the flow velocity in planes perpendicular to the free-stream direction. Over flat terrain, the wind-turbine wake was seen to slowly approach the ground while it propagated downstream. When introducing hilly terrain, the downward wake deflection was enhanced in response to flow variations induced by the hills, and the turbulent kinetic energy content in the wake increased due to the speed-up seen over the hills. The combined wake observed behind two streamwise aligned turbines was more diffused and, when introducing hills, it was more prone to deflect towards the ground compared to the wake behind an isolated turbine. Since wake interactions are common at sites with multiple turbines, this suggested that it is important to consider the local hill-induced velocity variations when onshore wind farms are analysed. Differences in the flow fields were seen when introducing either homogeneous or sheared turbulent inflow conditions, emphasising the importance of accounting for the prevailing turbulence conditions at a given wind-farm site to accurately capture the downstream wake development.
\end{abstract}

Copyright (C) 2017 John Wiley \& Sons, Ltd.

\section{KEYWORDS}

Wind turbines, Complex terrain, Wind-tunnel measurements.

\section{Correspondence}

E-mail: segalini@mech.kth.se

Received ...

\section{INTRODUCTION}

Wind turbines installed at onshore sites with complex terrains are often exposed to flow environments that greatly differ from those found at offshore locations. Mountainous terrains can introduce significant horizontal variations in the flow on 

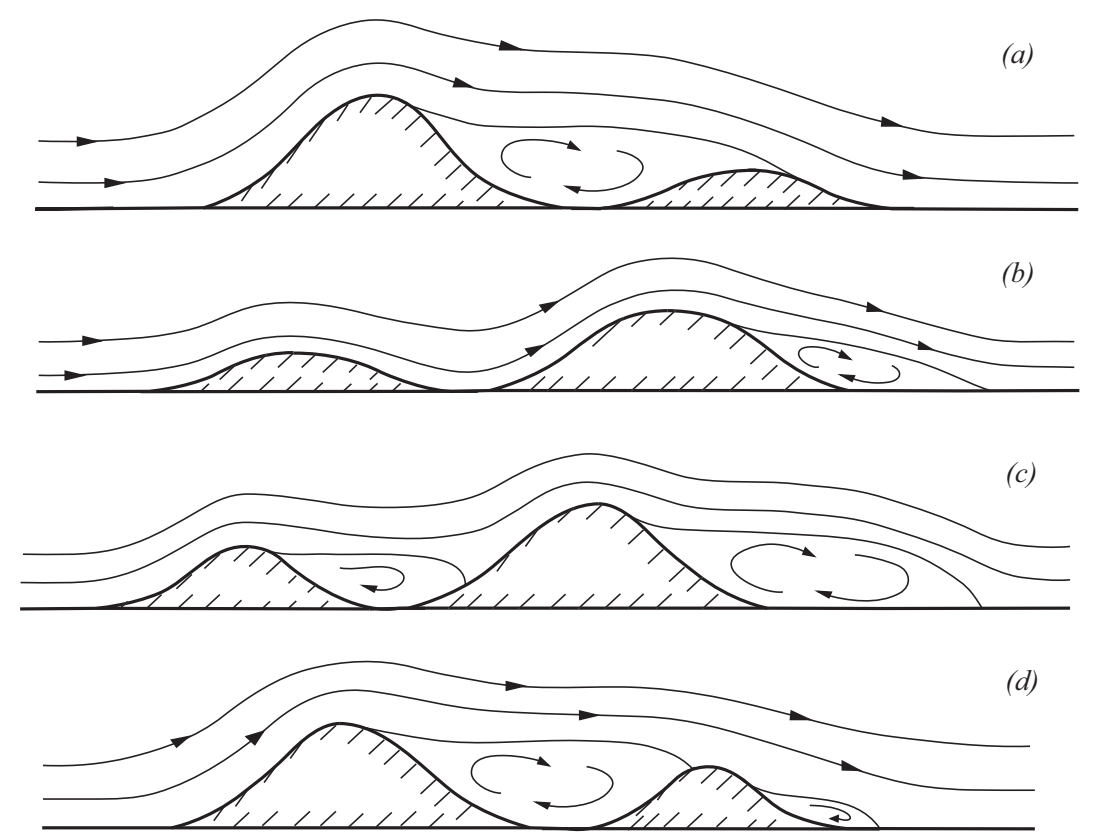

Figure 1. Sketch of the different flow fields that can arise from the combined effect of two hills with different heights, adopted from Kim et al. 7].

a large scale, since tall mountains may extend well beyond the reach of the atmospheric boundary layer [1]. The presence of a hill or a mountain ridge can also increase the turbulence levels in the flow, yielding higher power and load fluctuations for turbines installed at such sites [2]. The prevailing flow conditions at a complex-terrain site must therefore be carefully considered in order to avoid errors in the predicted wind-power availability [3].

Even a shallow hill can give rise to flow accelerations and decelerations that alter the typical logarithmic behaviour of the wind profile observed over a flat surface. This was for example seen during wind-tunnel measurements by Webster $e t$ al. [4], who observed the formation of internal boundary layers at inflection points where the hill geometry shifted from a convex to a concave curvature. Above the thin internal boundary layer (which typically reaches to about $1-2 \%$ of the hill half-width), the streamwise flow is generally more uniform, with less wind shear compared to a corresponding flat-terrain case [1]. The flow uniformity is particularly pronounced over hill crests, something that has been noticed in many previous studies [5, 6, 7, 8].

A theoretical model of the flow over low hills was developed by Jackson \& Hunt [9]. In their work a potential flow layer far from the hills was matched with the flow in a lower layer where the advection, pressure gradient and turbulent diffusion balance, leading to a parabolic differential equation. It is well known that linearised models work well as long as flow separation is absent, something that depends on the hill height but also on the hill geometry: a sharp hill crest can for instance trigger the flow to separate on its leeward side, yielding a more turbulent flow and reduced flow velocities in the downstream wake region. Regions with separated flows will also appear behind steep hills where the flow is no longer attached. As seen in the work by Arya et al. [5] and in the review by Finnigan [10], the flow over an isolated hill with a Gaussian or sinusoidal shape is likely to separate if the maximum slope of the hill is over $20^{\circ}$, whereas shallow hills with slopes less than $10^{\circ}$ are unlikely to cause flow separation. Thin separated flow regions with intermittent separation lines have been noted for hill slopes with intermediate values.

In landscapes featuring several hills, the final flow behaviour will be determined by the combined hill geometry. As illustrated in Figure 1a, a large upstream hill may submerge a lower hill in its wake. In contrast, the smaller hill will have a lower impact on the final flow field in a reversed flow situation, given that no flow separation occurs on its lee side (see Figure 13). As discussed by Røkenes and Krogstad [8], the speed-up seen over a downstream hill is heavily reduced if a recirculation region with massive flow separation exists on the leeward side of a nearby upstream hill. This is perhaps 
one of the most crucial aspects to consider during wind-farm siting in highly-complex terrains. The momentum losses behind a large upstream hill may on some occasions reduce the wake extent of a nearby hill in its immediate proximity. An illustration of this effect, which for instance has been observed by Kim et al. [7] and Ferreira et al. [11], is given in Figure 1 -d, where the wake of the small hill is reduced in the latter case, due to the presence of the larger upstream hill.

If the hilly geometry is extended to a series of sinusoidal hills, the changes in the streamwise flow variations typically reach a constant periodic behaviour at a certain point (typically after the third hill). This was for instance observed during the wind-tunnel measurements by Miller \& Davenport [6] and Carpenter \& Locke [12]. Results from their studies indicate that the flow over the first hill will be similar to that over an isolated hill: A flow acceleration with a maximum speed-up near the hill top will occur on its upstream side, while the flow velocity will decrease and a small separated flow region will form on its leeward side. The separated flow region behind the first hill will reduce the velocity and increase the turbulence intensity at the second hill crest. A smaller separated-flow region can then be expected in the valley after the second hill, in accordance with the flow scenario illustrated in Figure 1 d. After this, a higher flow velocity can again be anticipated at the third hill top, though the velocity magnitude seen at first hill crest will not be reached. Milder flow variations can thereafter be expected over the subsequent hills. Note that these flow variations will be more pronounced near the surface of the hills, while the flow variations in the above-lying region will be significantly smaller.

When compared to an undisturbed case over flat terrain, a wind turbine placed behind one of the hill configurations in Figure 1 would feature a loss in power, due to the reduced inflow velocity it would perceive. Higher turbulence levels can also be expected behind the hills, something that increases the fatigue loads on the turbine, but also reduces the extent of the near-wake region and promotes the turbine wake recovery [13, 14, 15]. The faster wake recovery is beneficial in cases where the wakes behind multiple wind turbines interact [16 17] regardless of the local terrain topography. Similar to the wake effects seen behind a hill, the wake interference from an upstream wind turbine reduces the available wind speed and thereby degrade the performance of downstream turbines, although this effect decreases with increased turbine spacings 17 18.

As seen from the examples given above, favourable conditions for wind energy are often found on hill tops, where the speed-up effects due to the presence of the hills increase the power output of the turbines. Downstream of the hill crest, the perturbations caused by the topology may act both in favour or disadvantage for the turbines, depending on how the wind-turbine wakes are deflected by the site-specific terrain features, and whether the flow separates. In some complex-terrain situations, turbines placed at downwind hill slopes can perform better due to the vertical shift introduced between the rotors, while the opposite has been noted for turbines placed on upwind slopes [19 20, 21 22]. The actual flow at a site with large terrain variations is ultimately unique, and might not only depend on the local topographical situation, but also on terrain features upstream of the investigated wind site [23, 24]. The evolving climate conditions and the diurnal cycle of the atmospheric boundary layer will introduce additional variations in the flow, which will be seen in the data obtained from field measurements [25, 26], but the uniqueness of the prevailing flow inhibits comparisons between data measured at different complex-terrain sites. Sometimes, better insights regarding fundamental flow phenomena and turbine behaviours can therefore be gained by studying idealised terrain situations. This is often done by means of smallscale wind-tunnel testing or through numerical simulations [27, 28]. A downside of wind-tunnel testing is that full-scale wind turbines generally are too large to achieve dynamical similarity through matching of the Reynolds number. While this remains a concern in the community, studies by Chamorro et al. [29] have indicated that the mean velocity statistics in the wind-turbine wake become independent of Reynolds number (based on the velocity at hub-height and the rotor diameter) at $\operatorname{Re} \approx 4.8 \cdot 10^{4}$, and higher order statistics at $\operatorname{Re} \approx 9.3 \cdot 10^{4}$.

While the wake development of a wind-turbine over flat terrain has been well documented [30, 31], the list of terrain combinations that can potentially alter the flow around a wind turbine is nearly endless. One aspect of complex-terrain flows concerns how much the far-wake development of a turbine is influenced by flow accelerations and decelerations introduced by variations in the local terrain. This is something that has not been extensively studied in the past, especially when multiple hills and turbines are concerned. The purpose of the present study is therefore to introduce several periodic hills and to characterise the wake development downstream of a wind turbine, with and without the presence of the hilly 
terrain. In this way the alterations induced by local terrain features could be assessed. The chosen surface geometry, which consisted of a series of sinusoidal hills aligned in the streamwise direction, is similar to terrain models studied by Miller \& Davenport [6] and Carpenter \& Locke [12]. Another objective of the present work is to study how wake interactions influence the wake propagation under the different flow conditions. For this reason a second turbine was introduced, and the combined wake of two tandem wind turbines was compared to the single-turbine cases. Wind-tunnel measurements were conducted, using stereoscopic particle image velocimetry (PIV) and hot-wire anemometry. Additionally, measurements were made with an artificially-generated shear layer to evaluate how inflow shear and free-stream turbulence would alter the results.

The present paper is structured as follows: section 2 describes the setup used during the experimental campaign. Thereafter, results obtained during the measurement campaign with turbines and hills are shown in section 3 The presented results highlight differences in the flow, in terms of mean streamwise velocity and mean turbulent kinetic energy, when changing the test-section setup. Finally, section 4 summarise the findings made in the present work.

\section{EXPERIMENTAL SETUP}

The experimental campaign was conducted in the closed loop Boundary Layer (BL) wind tunnel at KTH [32]. The tunnel is a high flow-quality facility with low turbulence intensity (below $0.04 \%$ ), and it has a $4 \mathrm{~m}$ long test section with a 0.75 $\mathrm{m}$ high and $0.5 \mathrm{~m}$ wide cross-section area. The wind-tunnel velocity at the test-section entrance was held constant at 7.5 $\mathrm{m} / \mathrm{s}$ throughout the measurement campaign and it was monitored by using a Prandtl tube connected to a Furness pressure transducer. During the experiments, the first part of the test section was dedicated to the generation of an artificial inflow meant to introduce shear and free-stream turbulence, mimicking the conditions of an atmospheric boundary layer. The boundary layer was generated by means of a vertical grid followed by a row of triangular spires [33] and an aluminium diamond surface mesh that enhanced the turbulent activity close to the ground.

Previous measurements [34], indicated that the spires and grids induced a fairly linear shear in the region of interest around the turbine rotors, and stronger turbulent fluctuations when approaching the ground. This can be seen in Figure 2 where the mean streamwise inflow velocity and the normalised velocity standard deviation are shown at a plane downstream of the spires and grid (the streamwise position of this plane is indicated with the annotation Inflow in Figure 3I). The free-stream velocity was in this case defined as the velocity measured at hub height downstream of the spires, giving $U_{\infty}=6.25 \mathrm{~m} / \mathrm{s}$ with the sheared inflow conditions. The results in Figure 2 have been averaged over the spanwise direction and the horizontal bars indicate the spanwise variability of the flow (the standard deviation of the mean velocity in the spanwise direction). The origin of the vertical $Z$-coordinate in the figure (normalised with the rotor diameter, $D$, of the wind-turbine models used) is at hub height and the ratio of the rotor diameter to the test-section height, $H$, is $D / H \approx 0.15$.

The hot-wire measurements were made by means of constant-temperature hot-wire anemometry, using a single-wire probe and a Dantec Streamline Pro system. The vertical velocity profiles were measured at five evenly distributed spanwise stations, where each profile was measured at 20 points with a $12 \mathrm{~mm}$ vertical distance between the measurement points. At each point, the flow velocity was measured during 40 seconds at a sampling rate of $10 \mathrm{kHz}$. The hot-wire probe was calibrated before and after the measurements to ensure that no drift had occurred. The experimental uncertainty of the averaged velocity (measured with the hot-wire probe) was $\epsilon_{U}=1.6 \%$ with a $95 \%$ confidence interval.

The second half of the test section was used for the terrain model, which consisted of five sinusoidal hills aligned in the streamwise direction. The first hill was placed about $17 D \approx 1.9 \mathrm{~m}$ downstream of the spires and the hill height was given by the expression $h / D=0.31[1-\cos (\pi x /(2 D))]$. The hills were $70 \mathrm{~mm}$ high and made of XPS styrofoam. The hill periodicity was $4 D=448 \mathrm{~mm}$ and the maximum slope of the hills was about $17^{\circ}$, meaning that a small region of separated flow could be expected in the hill valleys [5, 10]. The surface of the hills was covered with a layer of black adhesive plastic to avoid laser reflections during PIV measurements: this also ensured that the surface of the hills was fairly smooth. The hill height was decided with the aid of CFD simulations considering both wind-tunnel blockage effects and the speed-up 

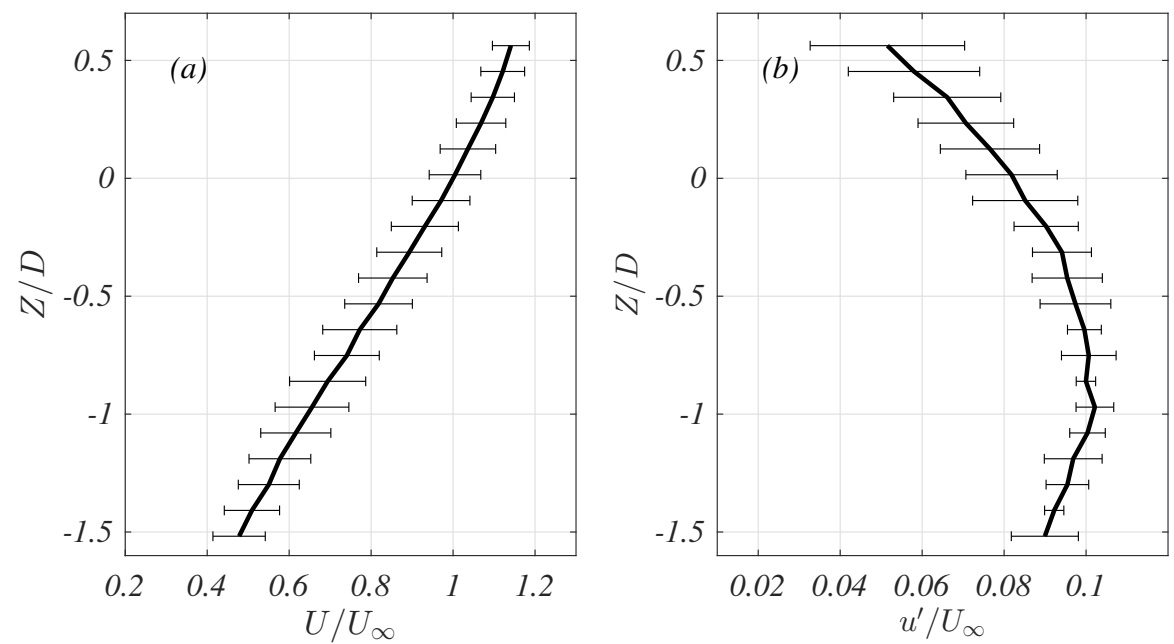

Figure 2. Vertical inflow profiles with spires and grids in terms of the mean streamwise velocity $(a)$ and velocity standard deviation (b). The horizontal bars indicate the variability in the spanwise direction. The streamwise location where the profiles were measured is shown in Figure $3 t$.

induced by the hills. With the chosen hill height, a $11 \%$ speed-up (at hub height) was estimated when including the windtunnel roof, compared to a $7 \%$ speed-up in the unblocked situation. The blockage ratio of the wind-turbine rotors projected on the test-section cross plane minus the hills frontal area was $3.2 \%$ : therefore, blockage effects from the turbines were assumed to be negligible.

To distinguish between the effect of the different elements introduced in the present setup, multiple measurements were performed with varying test-section configurations. The reference configuration was without hills, spires and grids, and it will be referred to as the plain configuration in the following. The second configuration (referred to as the hills configuration) included hills but no spires and grids. Finally, the configuration with hills, spires and grids will be denoted as the hills+spires configuration. For all three configurations, measurements were made both with a single turbine and multiple ones. During the single-turbine cases, the turbine was placed upon the ridge of the second hill. Then in a similar manner, two turbines were used in the multiple-turbine cases, and these were placed on the ridges of the first and second hill. In all cases, the turbines positions were at the tunnel spanwise centreline. A sketch of the measurement setup is shown in Figure 3 , including the positions of the turbines and the normalised streamwise coordinates of the measurement planes monitored with the various flow configurations. It is also worth to underline that when studying the plain configuration, $70 \mathrm{~mm}$ long adapters were attached to the towers in order to keep the vertical distance between the turbines and the PIV cameras fixed compared to the hills configuration. Therefore, the absolute distance of the turbine from the ground was greater in the plain case compared to the hill case, so that the plain case could be considered more similar to an isolated turbine.

With the plain configuration, a uniform inflow velocity of $U_{\infty}=7.5 \mathrm{~m} / \mathrm{s}$ and a streamwise turbulence intensity below $0.04 \%$ was measured over the region spanned by the rotor (using hot-wire anemometry) [34]. The boundary layer developing over the flat surface could thereby be presumed to have a small influence on the turbine wake, at least in the near-wake region of the turbine. The streamwise and the vertical velocity profiles (measured through PIV) along the tunnel centreline, for the flow configurations with hills and hills+spires, without the presence of wind turbines, are given in Figures 45 The origin of the vertical coordinate is located at the turbine hub height $(1.43 \mathrm{D}$ from the surface of the hill-top) and the streamwise positions of the profiles are shown in Figure $3 I$. For all configurations, the free-stream velocity $U_{\infty}$ was defined as the one measured at hub height far upstream of the first hill.

As seen from Figure 4 the hills give rise to a fairly uniform inflow in the rotor-region, with a speed-up around 6-8\%. With the hills+spires configuration, the vertical shear seen over the hills in Figure 4 is consistent with the inflow profile 

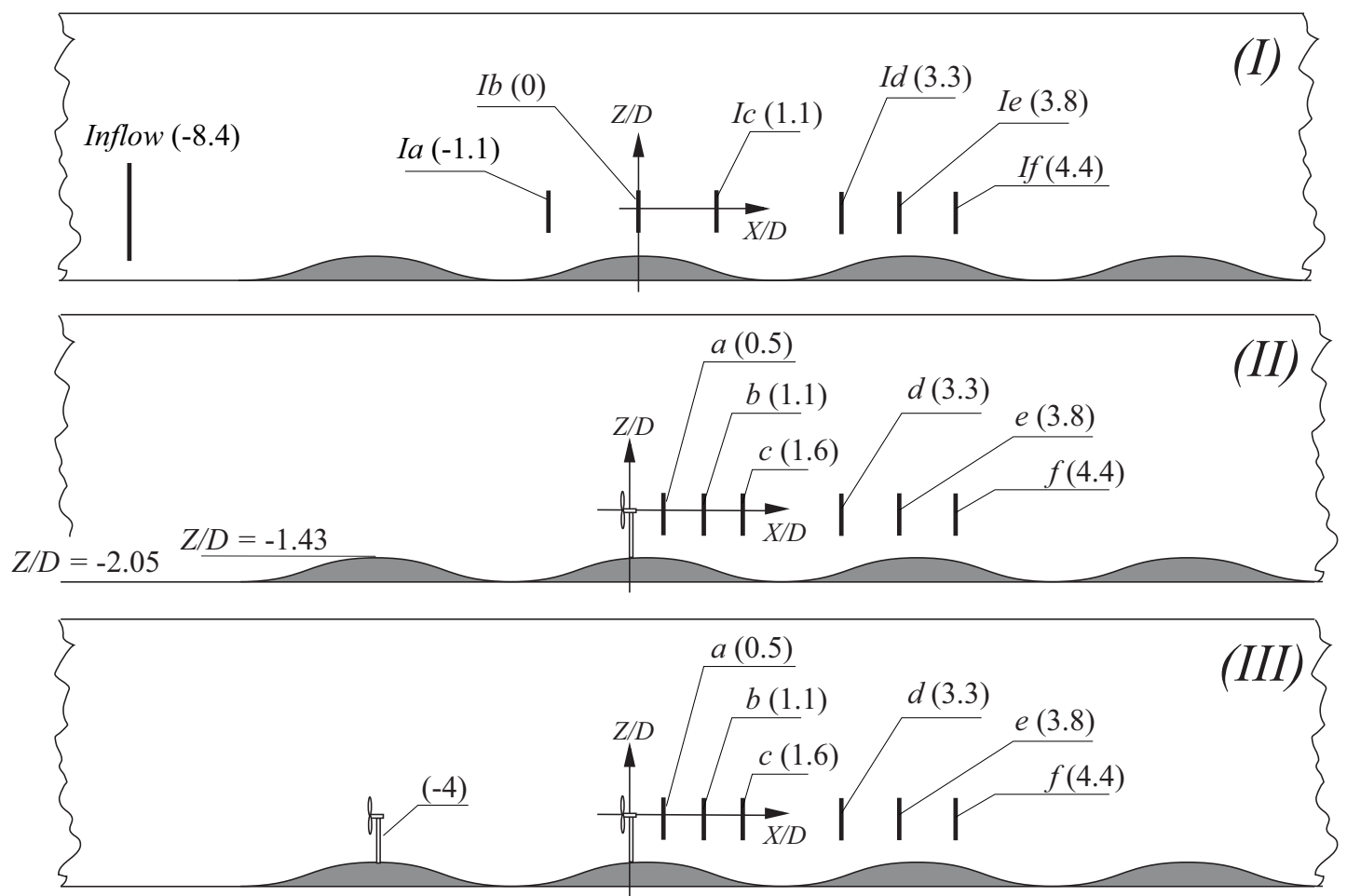

Figure 3. Schematic representation of the test-section setup for the flow configurations without turbines (I), with one turbine (II) and with two tandem turbines (III). The origin of the coordinate system used during the measurement analysis (normalised with the rotor diameter $D$ ) is also indicated. For each measurement configuration, the locations of the analysed measurement planes are indicated, and their normalised streamwise coordinates $(X / D)$ are given inside the brackets. For the flow configurations without hills, the distance between the rotor and the test-section floor was unchanged compared with the illustration.
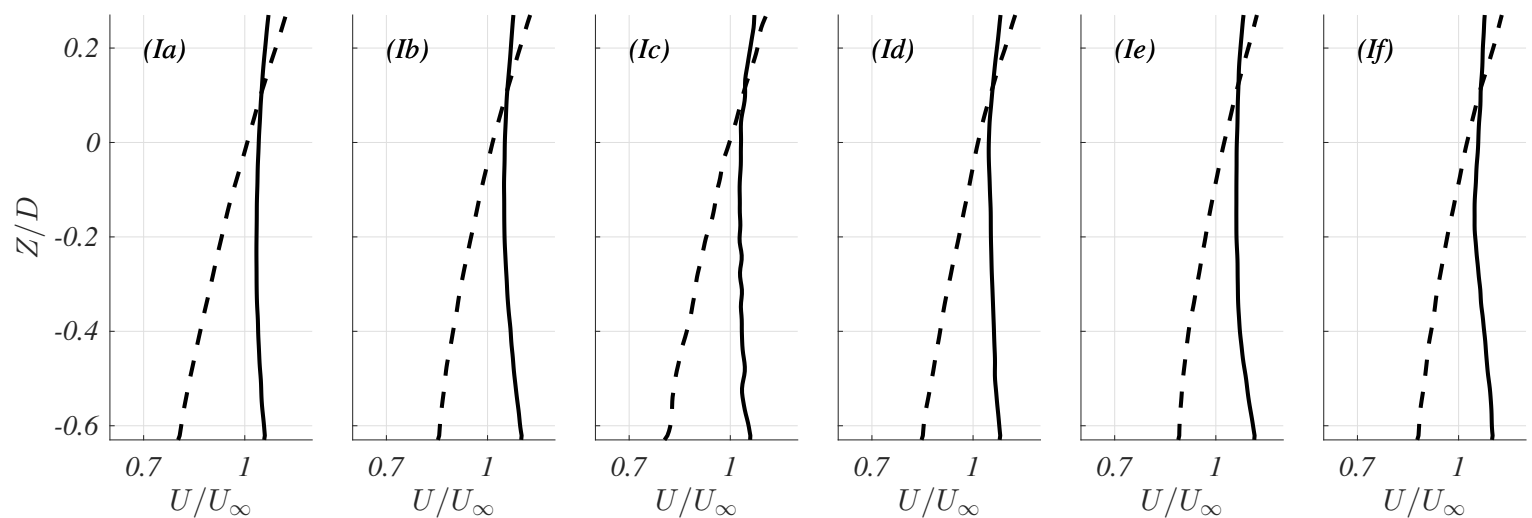

Figure 4. Normalised streamwise velocity, $U / U_{\infty}$, along the tunnel centreline for the configuration with hills (solid lines) and hills+spires (dashed lines). The streamwise positions of the profiles (in la-lf) are given in Figure 3 .

reported in Figure 2, although some small variations can be seen in the lower rotor region, possibly due to the presence of the hills. The vertical flow variations seen in Figure 5 confirm that the hills give rise to flow perturbations in the order of $\pm 5 \%$ of $U_{\infty}$. These are particularly pronounced over the downwind slopes, indicating that a vertical transfer of momentum takes place in these regions in order to replenish momentum losses over the leeward hill sides. The vertical flow variations are somewhat reduced with the hills+spires configuration, suggesting that the stronger turbulent mixing seen with the sheared turbulent inflow conditions weakens the flow perturbations induced by the hills. 

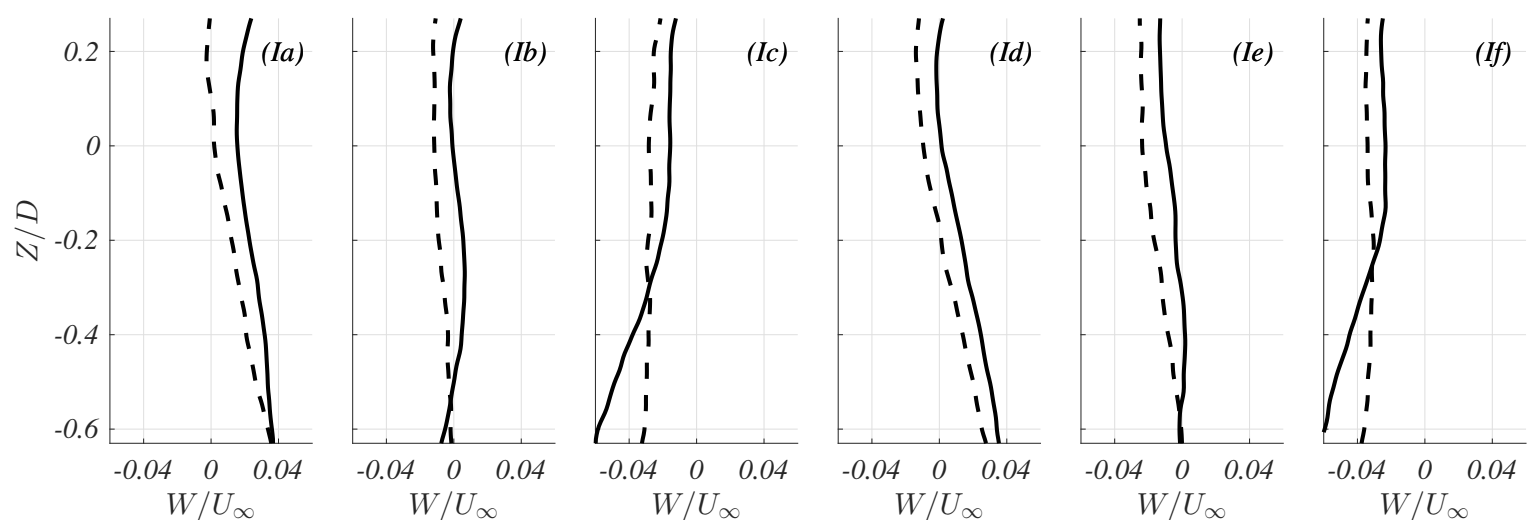

Figure 5. Normalised vertical velocity, $W / U_{\infty}$, along the tunnel centreline for the configuration with hills (solid lines) and hills+spires (dashed lines). The streamwise positions of the profiles (in la-If) are given in Figure 3 .

Table I. Airfoil parameters for the rotor used during the measurements. The first profile at $z=10 \mathrm{~mm}$ is at the root chord while the last profile at $z=54 \mathrm{~mm}$ is $2 \mathrm{~mm}$ from the the blade tip.

\begin{tabular}{lcccccccc}
\hline$z[\mathrm{~mm}]$ & $t[\mathrm{~mm}]$ & $x_{L E}[\mathrm{~mm}]$ & $y_{L E}[\mathrm{~mm}]$ & $\Delta x[\mathrm{~mm}]$ & $a_{0} \cdot 10^{4}[\mathrm{~mm}]$ & $a_{1} \cdot 10^{2}[-]$ & $a_{2} \cdot 10\left[\mathrm{~mm}^{-1}\right]$ & $a_{3} \cdot 10^{3}\left[\mathrm{~mm}^{-2}\right]$ \\
\hline 10 & 1.05 & 0 & 0 & 19.57 & -3 & 2.99 & -8.44 & -0.8 \\
19 & 0.95 & 0.72 & -0.43 & 17.65 & -4 & 3.06 & -7.49 & -36.7 \\
28 & 0.78 & 2.0 & -1.3 & 15.56 & -2 & 2.54 & -5.91 & -3.1 \\
37 & 0.66 & 2.77 & -1.67 & 13.6 & -2 & 2.53 & -4.82 & 8.8 \\
46 & 0.62 & 3.34 & -1.82 & 11.59 & -2 & 2.65 & -3.85 & 5.7 \\
54 & 0.54 & 5.1 & -1.88 & 9.54 & -38 & 7.73 & -4.38 & -55 \\
\hline
\end{tabular}

\subsection{Wind-turbine models}

The wind-turbine rotors used during the measurements were made of carbon-fibre reinforced plastic, with thin-bladed slightly cambered airfoils that had a fixed pitch angle and diameter $D=112 \mathrm{~mm}$. The rotor airfoils had a constant chord thickness and the mean camber line of the airfoil profiles could be described by third-order polynomials on the form $y=y_{L E}+\sum_{n=0}^{3} a_{n}\left(x-x_{L E}\right)^{n}$ where the $L E$ subscript denotes the $x$ and $y$-offset from the leading edge of each airfoil. In Table $\mathbb{1}$ the constants $a_{n}$, the chord thickness, $t$, the spanwise airfoil position $z$ and the offset to the trailing edge (TE), $\Delta x=x_{T E}-x_{L E}$, are given for a number of airfoils along the blade length. In this case, a right-handed coordinate system was used, where the $z$-coordinate was set along the span of the rotor blade and the $y$-coordinate was aligned with the rotor axis.

The rotor had a Reynolds number $\mathrm{Re} \approx 6 \cdot 10^{4}$ based on the rotor diameter and the velocity at hub height. This was close to the values reported by Chamorro et al. [29] for achieving Reynolds-number independency. Each rotor was connected to a Faulhaber DC-micro motor that worked as a generator, which made it possible to measure the torque of the turbines through the relationship $Q=k_{1} I+k_{2}$, where $k_{1}$ is a torque constant and $k_{2}$ is the friction torque of the generator. The constants were calibrated for each generator before the start of the measurement campaign. The calibration of the DC motors was done by means of an aluminium disc with a known inertia, which was accelerated by the DC motor up to a certain rotational velocity. The electrical circuit of the DC motor was then switched so that the motor operated in a generator mode and the DC-motor voltage output and the angular velocity, $\omega$, of the disc were monitored while the disc decelerated. During the deceleration process, the mechanical torque of the disc could be estimated by $Q=J \dot{\omega}+0.616 \cdot \pi \rho r^{4} \sqrt{\nu \omega^{3}}$, where $r$ is the radius of the disc. The first term in this equation represents the inertia of the disc while the second term corresponds to the aerodynamic drag of the rotating disc, assuming a laminar flow regime and neglecting edge effects [35]. The Reynolds number at the edge of the disc (based on the angular velocity during the calibration and the disc radius, $r=50 \mathrm{~mm}$ ) was $\operatorname{Re} \leq 252$, which was below the critical Reynolds number for turbulent transition, $\operatorname{Re}_{c} \approx 500$, typically seen for rotating 
discs [36], justifying the laminar-flow assumption made in the torque estimation. From the acquired calibration data, the torque constant, $k_{1}$, and the friction torque, $k_{2}$, of the DC motors could be found by means of a least-squares technique.

The angular velocity of the turbines could be adjusted by changing the electrical load applied on the generators. During the measurements, the electrical load was held constant to the value associated to the maximum power coefficient for each turbine. The generators were attached to $16 \mathrm{~cm}$ tall aluminium towers and the angular velocity, $\omega$, of the turbines was measured by means of a laser and a photodiode detecting the blade passages. The turbine power was then calculated as $P=\omega Q$. Over flat terrain, the wind-turbines had a maximum power coefficient of $C_{P} \approx 0.36$ at a tip-speed ratio $\lambda=3$, defined as $C_{P}=P /\left(\frac{1}{2} \rho U_{\infty}^{3} A_{D}\right)$ and $\lambda=\omega R / U_{\infty}$, respectively, with $A_{D}$ as the projected rotor area and $R$ as the rotor radius. The experimental uncertainty of the measured torque and angular velocity was $\epsilon_{Q}=1.2 \%$ and $\epsilon_{\omega}=2 \%$, respectively, while the free-stream velocity, $U_{\infty}$, could be measured with a $0.1 \%$ uncertainty. The thrust, $T$, of the turbines was also measured by means of a force balance consisting of four strain gauges. These were attached to one of the turbine towers and mounted into a full Wheatstone bridge configuration. The thrust-voltage relationship was found through a calibration procedure by using known reference weights. The thrust coefficient of the turbine could then be calculated as $C_{T}=T /\left(\frac{1}{2} \rho U_{\infty}^{2} A_{D}\right)$. The thrust coefficient of the turbine was about $C_{T} \approx 1.2$ at a tip-speed ratio of $\lambda=3$. This value is higher than the thrust coefficient commonly expected for a full-scale turbine operating under optimal conditions, likely due to a region with flow recirculation observed immediately behind the turbine. This suggested that the wake flow was representative of a heavily-loaded rotor. Further information about the turbine thrust and power performance under different flow configurations, and with varying levels of wake interaction, can be found in a preceding study [34].

\subsection{PIV measurements}

When performing PIV measurements in the wind tunnel, DEHS oil was injected as smoke and used as seeding particles in the flow. The injected particles were illuminated by a laser sheet in two rapid pulses, with a $100 \mu$ s time delay, that were captured from two different angles through two high-speed cameras. A LaVision calibration target was used for adjusting the positions of the cameras and to perform image calibrations. Additional image calibrations were made through disparity mapping for final enhancements. The plane illuminated by the light sheet was around $2 \mathrm{~mm}$ thick and perpendicular to the free stream. Its distance from the turbines was controlled using an Isel traversing system, which shifted the rigidly joined laser and camera system in the streamwise direction. A Litron Nano-PIV pulsed Nd:YAG laser was used as light source, emitting light pulses of $600 \mathrm{~mJ}$ at a wavelength of $532 \mathrm{~nm}$, and a spherical and a cylindrical lens were used to generate the light sheet. The laser was controlled by using a LaVision PTU X high-speed controller unit that also controlled two high-speed 10-bit CMOS cameras of type Photron Fastcam APX RS. The cameras captured image pairs at $50 \mathrm{~Hz}$ for 61.44 seconds and they were equipped with lenses that had a focal length of $105 \mathrm{~mm}$. The PIV system was controlled using the LaVision Davis 8.3 software, which was also used for processing to reconstruct instantaneous flow fields from the particle images.

Constraints related to the wind-tunnel dimensions and the lenses focal distance limited the field of view to $10 \mathrm{~cm} \times$ $12 \mathrm{~cm}(0.9 D \times 1.1 D)$ during the measurements: the centre of the measurement planes was for this reason fixed to a point slightly below hub height. This was believed to be the best approach for following the wake as it propagated downstream. The greatest impact from the presence of the hills was expected to occur in the lower shear-layer region of the wake, making this region more interesting to study instead of the area above the turbine hub height.

During the pre-processing, the average minimum intensity was first removed from the particle images by applying a sliding minimum filter (with an 8 pixel size) to remove background noise. Thereafter, stereoscopic reconstruction (based on cross-correlation) was performed on the images, using multiple passes with decreasing size, to reconstruct the instantaneous flow fields. The final interrogation windows used during the flow-reconstruction process had a size of $32 \times 32$ pixels, which gave a spatial resolution of $0.85 \times 0.85 \mathrm{~mm}^{2}$. The precision of the cross-correlation operation could be estimated to 0.1-0.5 pixel following the guidelines by Raffel et al. [37]. 

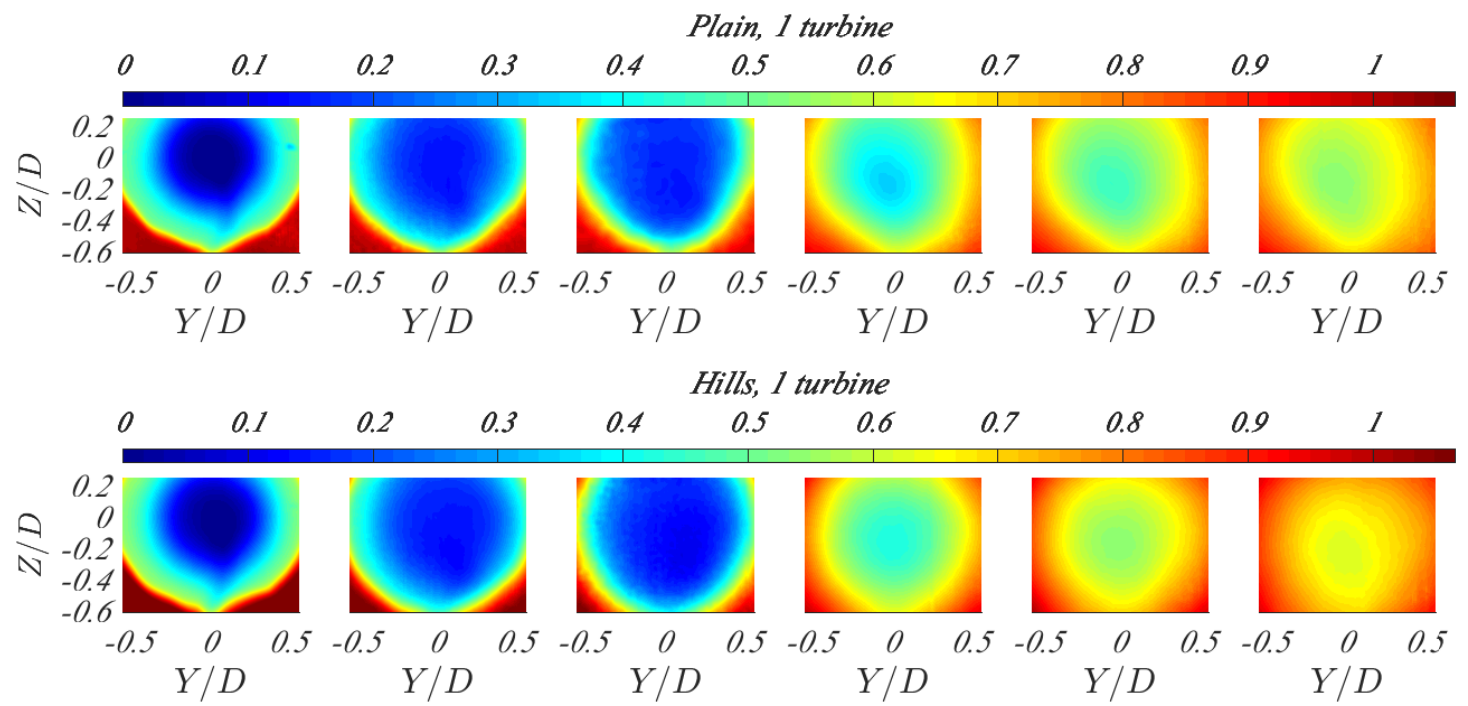

Figure 6. Normalised streamwise velocity, $U / U_{\infty}$, in the wake of one turbine without hills (top) and with hills (bottom). The planes (from left to right) are viewed at the streamwise locations (a-f) indicated in Figure $3 I$.
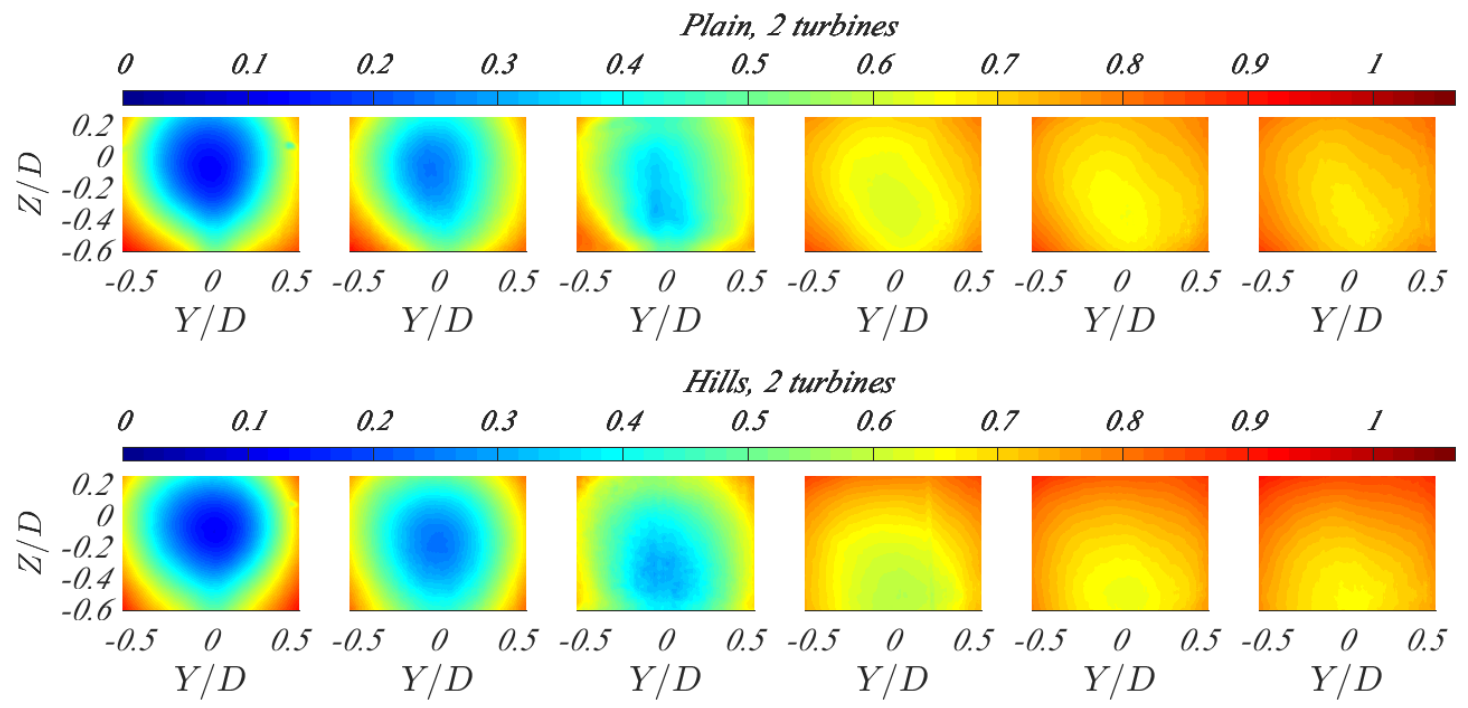

Figure 7. Normalised streamwise velocity, $U / U_{\infty}$, in the wake of two turbines without hills (top) and with hills (bottom). The planes (from left to right) are viewed at the streamwise locations (a-f) indicated in Figure 3 III.

\section{RESULTS}

The mean velocity variation behind a single turbine, with and without hills, is shown in Figure 6(the streamwise positions of the measurement planes are indicated in Figure $3 I I$ ). In both configurations, the wakes are slightly asymmetric in the vertical direction due to the presence of the turbine tower. It is further evident that, while the initial mean flow fields are very similar, the downstream wake diffusion is stronger with hills.

The mean flow field for the single-turbine cases (Figure 6) can be compared to the corresponding flow field with two turbines shown in Figure 7 When introducing a second turbine a more diffused wake can be observed, in agreement with results presented by Mycek et al. [16, 17] for wind turbines over flat terrain. This can be seen in more detail in Figure 8 which displays the mean streamwise velocity profiles along the centreline of the same planes, for the plain and hilly 

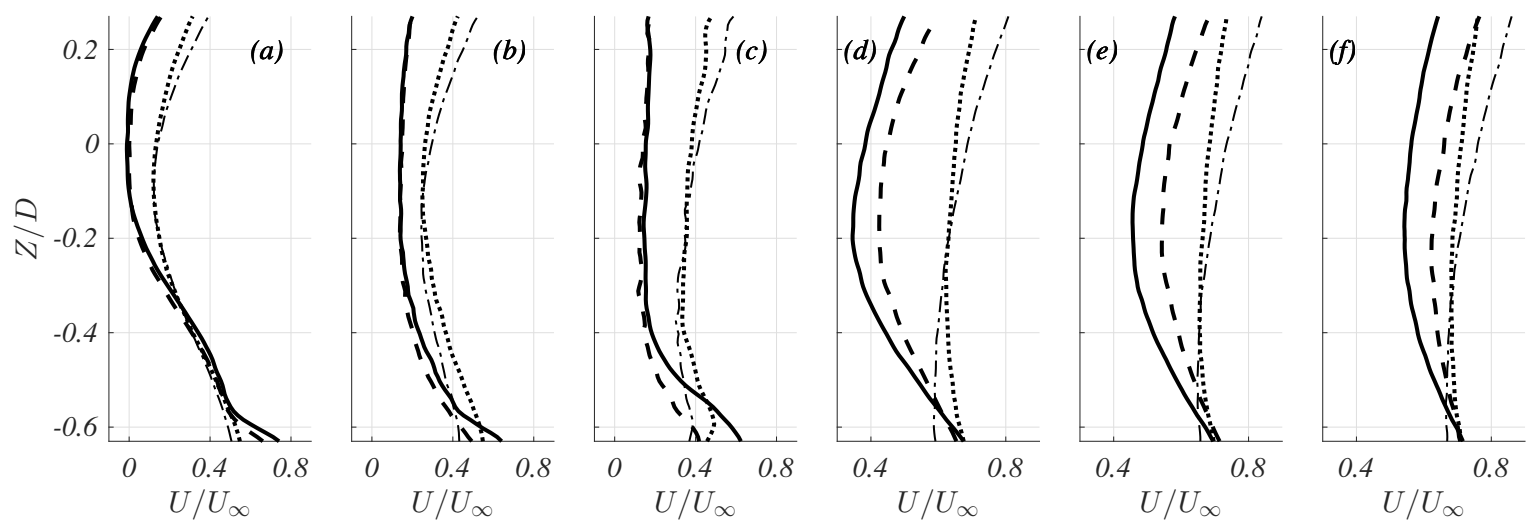

Figure 8. Normalised streamwise velocity, $U / U_{\infty}$, along the rotor centreline in the single-turbine wake with the configurations plain (solid lines) and hills (dashed lines), and in the tandem-turbine wake with the configurations plain (dotted lines) and hills (dasheddotted lines). The streamwise locations where the profiles were measured are shown in Figures $3 I I-I I I$. The last three graphs (d-f) have different velocity axes for better visualisation.

configurations. The results are shown both in the wake of the single turbine and behind the two tandem turbines. The faster wake recovery in the cases with hills is clearly distinguishable, particularly in the far-wake region of the turbines. In Figure 81-f, the difference between the single-turbine plain and hilly cases is most pronounced in the upper part of the studied flow region, where a higher flow velocity can be seen with hills, due to the higher wake diffusion. Closer to the ground surface, the velocity magnitude is instead quite similar for both single-turbine cases, as the outer region of the wake is approached. A clear difference between the velocity gradient in the plain and hilly case can furthermore be seen in the cases with two turbines. Above $Z / D>-0.2$, a higher streamwise velocity can be seen in the tandem-turbine wake with hills compared to the corresponding plain configuration, while the opposite is observed closer to the ground surface $(Z / D<-0.2)$. If the region with the lowest streamwise velocity is regarded as the wake centre, this suggests a downward shift of the wake in the case with hills, which could be caused by the stronger vertical velocity variations seen over the hills in the region where $Z / D<-0.2$ (see Figure 5).

Arguably, the turbine is on average closer to the ground in the cases with hills, which could partly explain the stronger downward deviation seen with this configuration. Effects from the wall-distance to the turbine hub-height (over a flat terrain) have for instance been seen by Dobrev and Massouh [31], although they noticed an increased upward wake deflection when the turbine was moved outside of the wall-boundary layer. However, the increased wake diffusion seen in the present work when introducing hills, was not observed in the flat-terrain study by Dobrev and Massouh [31]. Together with the observations in Figure 45 this suggests that the speed-up and the vertical velocity induced by the hills lead to a stronger turbulent mixing and, consequently, to a faster breakdown of the turbine wake.

The turbulent kinetic energy for the single-turbine case with hills and with the plain configuration is displayed in Figure 9 As seen in the figure, the speed-up over the hills gives rise to stronger turbulent fluctuations in the wake, which promotes the faster wake diffusion seen in Figure 8 . The leftmost planes in Figure 9 (captured 0.5D downstream of the turbine) show traces of both tip-vortex and root-vortex regions. Signatures of the tip-vortex region can clearly be seen up to $1.6 D$ downstream of the rotor (location $c$ in Figure $3 I I$ ), while the root-vortex region is no longer visible beyond $1.1 D$ downstream of the turbine (location $b$ in Figure $3 I I$ ). In the tandem-turbine wake (not shown here) the vortex regions were less distinct, due to the wake interaction between the upstream and downstream turbine.

For comparison, the turbulent kinetic energy distribution along the rotor centreline, for the hilly and plain cases, with one and two turbines, is shown in Figure 10 Higher turbulent kinetic energy levels are seen near hub height in Figure 10. for the single-turbine cases, due to root vortices and the wake behind the turbine nacelle. In the single-turbine wake, strong energy fluctuations are also seen near bottom-tip region of the rotor, where tip vortices intersect the measurement plane. By comparison, the combined wake from the two aligned turbines is from the beginning much more diffused, and the region with high turbulent kinetic energy near hub-height, corresponding to the passing of root vortices, is not present 

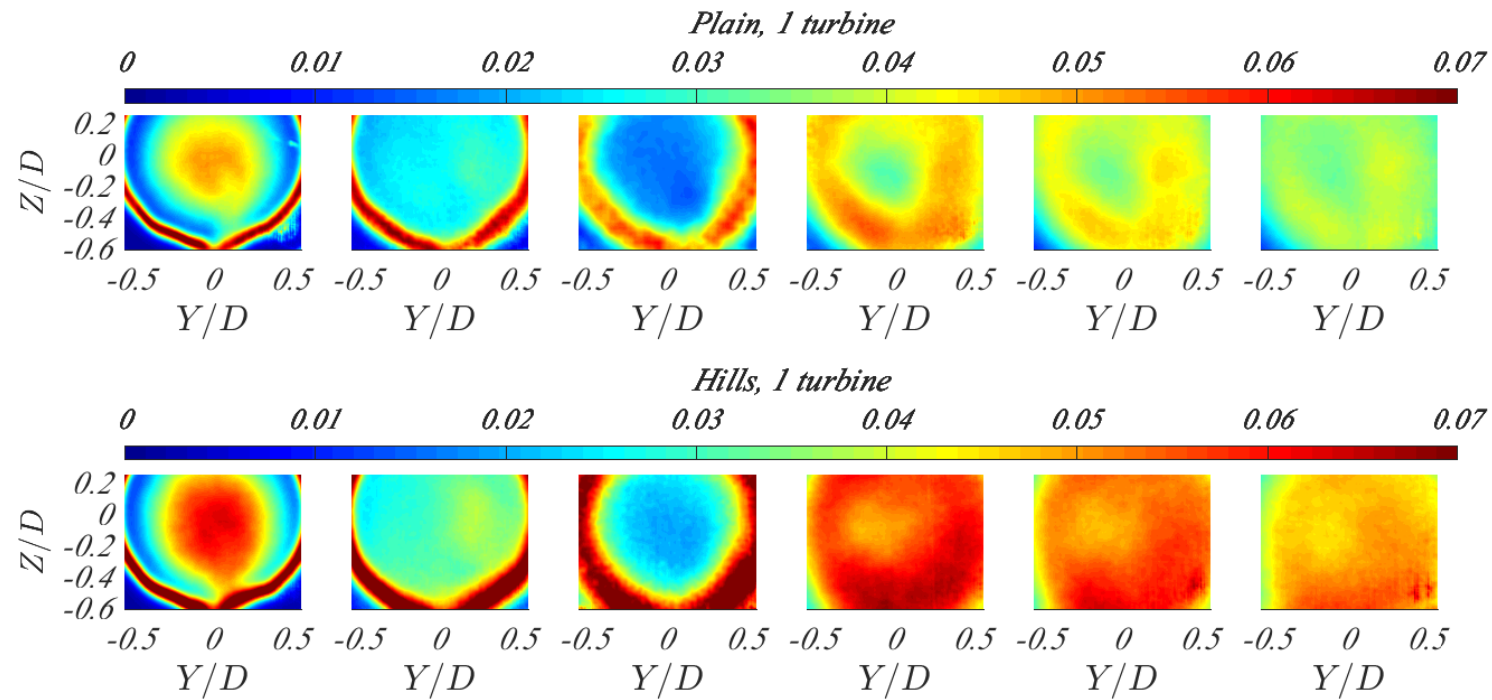

Figure 9. Normalised turbulent kinetic energy, $K / U_{\infty}^{2}$, in the wake of one turbine without hills (top) and with hills (bottom). The planes (from left to right) are viewed at the streamwise locations (a-f) indicated in Figure $3 I$.
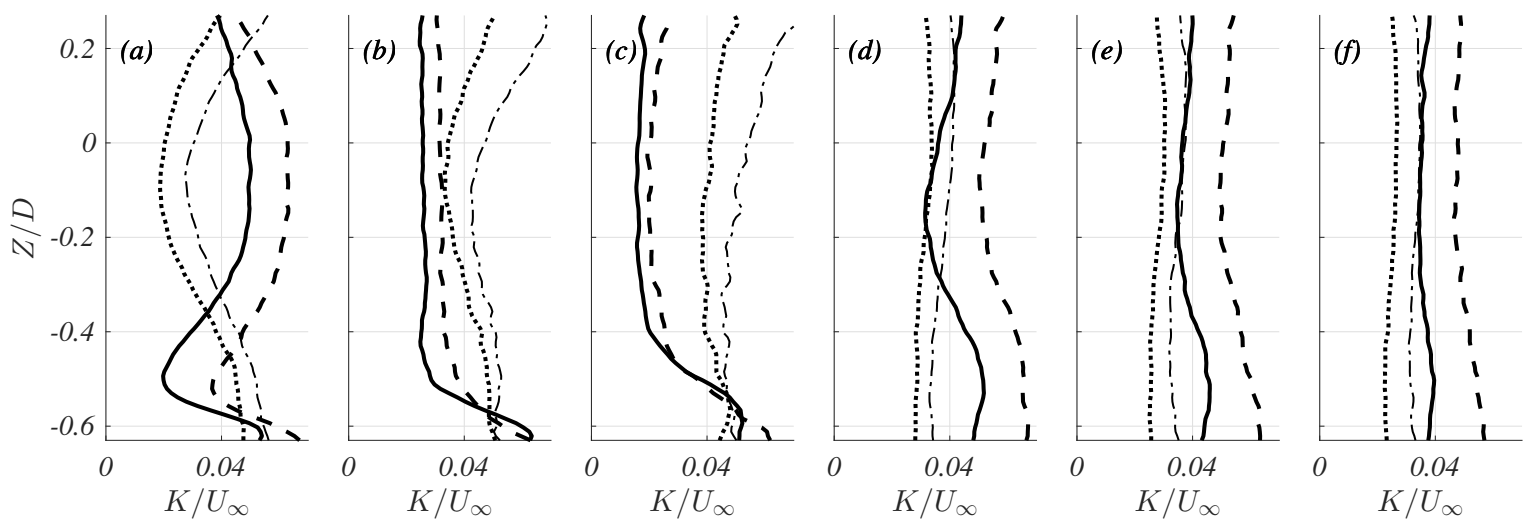

Figure 10. Normalised turbulent kinetic energy, $K / U_{\infty}^{2}$, along the rotor centreline in the single-turbine wake with the configurations plain (solid lines) and hills (dashed lines), and in the tandem-turbine wake with the configurations plain (dotted lines) and hills (dasheddotted lines). The streamwise locations where the profiles were measured are shown in Figures $3 I I-I I I$.

in the flow field in Figure 10 . However, a higher turbulent kinetic energy content can be seen near the blade-tip regions in Figure $10 \mathrm{a}-\mathrm{c}$, indicating that traces of tip-vortices remain some distance downstream of the rotor. In both the single and multiple-turbine cases, higher turbulent kinetic energy levels can be seen when introducing hills. Since the tandem-turbine wake is more diffused, with less organised vortical structures than the single-turbine wake, it is possible that the multipleturbine wake is more affected by the vertical flow variations seen in Figure 5 This could explain the somewhat higher wake deflection observed in the two most downstream planes in Figure 7 compared with the corresponding planes in the single-turbine case with hills in Figure 6

\subsection{The impact of the turbulent shear layer}

Figure 11 shows how the mean flow field in the wake behind one or two turbines changes when introducing spires and grids to mimick the effect of the atmospheric boundary layer (for better visualisation, the velocity scale has been changed in this figure compared to the homogenous-inflow cases in Figures 6 and 7 . For this configuration, hills were included in both the single- and multiple-turbine case. The main effect seen on the results is a more rapid wake diffusion compared to 

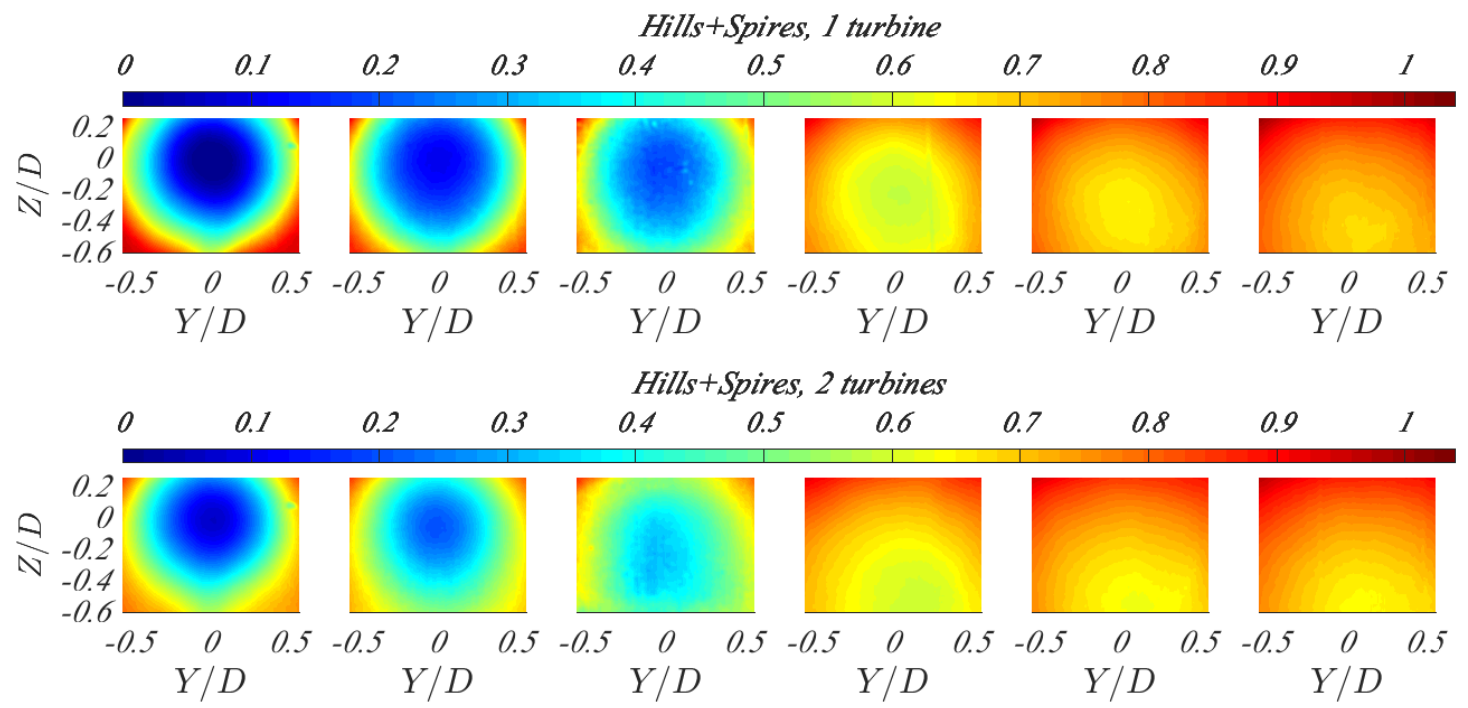

Figure 11. Normalised streamwise velocity, $U / U_{\infty}$, in the wake of one turbine (top) and two turbines (bottom), with the hills+spires configuration. Each plane (from left to right) is viewed at the streamwise locations (a-f) indicated in Figures $3 I I-I I I$.
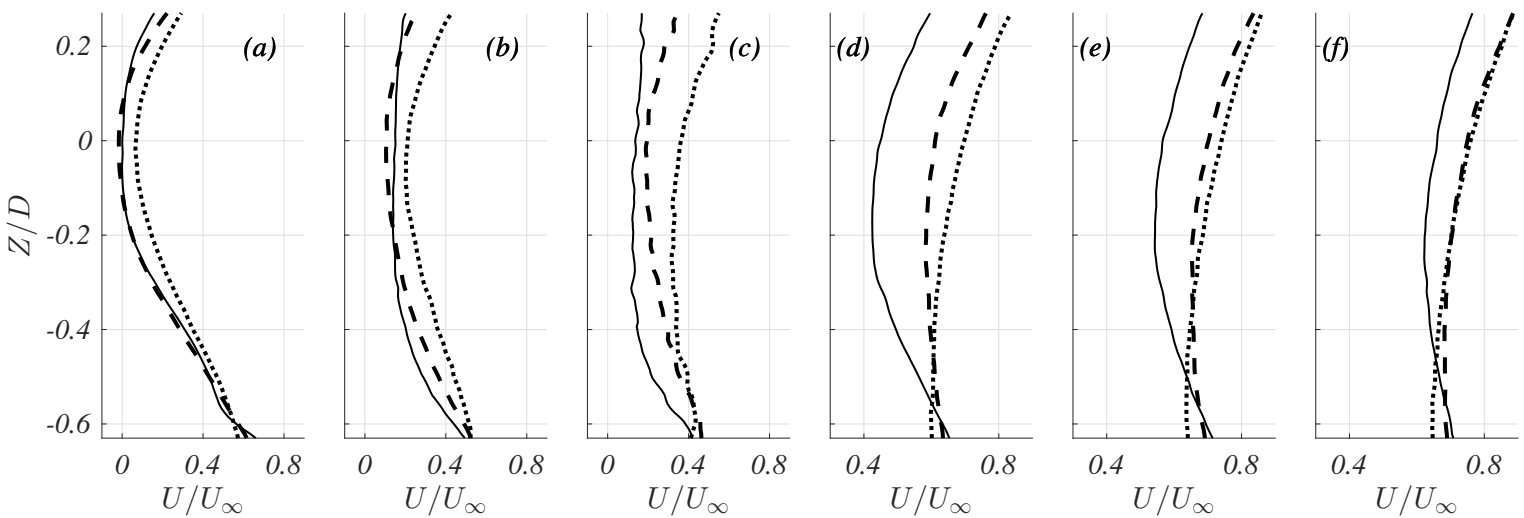

Figure 12. Normalised streamwise velocity, $U / U_{\infty}$, along the rotor centreline with the hills+spires configuration, with one turbine (dashed lines) and two turbines (dotted lines). As a reference, the single-turbine case with hills is also included (solid lines). The streamwise locations where the profiles were measured are shown in Figures $3 I I-I I I$. The last three graphs $(d-f)$ have different velocity scaling for better visualisation.

the homogeneous-inflow cases, due to the increased free-stream turbulence introduced in the flow field. Also, the wakes seem to deflect downwards as they propagate downstream. This is consistent with the results noted for the homogeneous inflow cases with hills, suggesting that the turbine wakes are influenced by the hills presence even when submerged in a turbulent shear layer. The mean velocity profiles along the rotor centreline for the hills +spires configuration, with one and two turbines, are shown in Figure 12 For comparison, the single-turbine profiles from the case with hills are included as a reference. Here, it is once more evident that the tandem-turbine wake diffuses faster than its single-turbine counterpart, even when high turbulence levels are present in the inflow to the first turbine. In both cases with the hills+spires configuration, the wake approaches a downstream velocity profile that resembles the sheared conditions seen at the inflow in Figure 2 . giving a visibly different velocity distribution in the far-wake region (Figure $12 \mathrm{~d}-\mathrm{f}$ ) compared to the single-turbine case with hills.

Regarding the turbulent kinetic energy in the wakes, the tip-vortex regions seen in Figure 13 are less pronounced than with the homogenous inflow (Figure 9), particularly close to the ground. Flow variations induced by the sheared inflow conditions can be distinguished in the kinetic energy fields, and an enhanced vortex breakdown can be seen, which is 

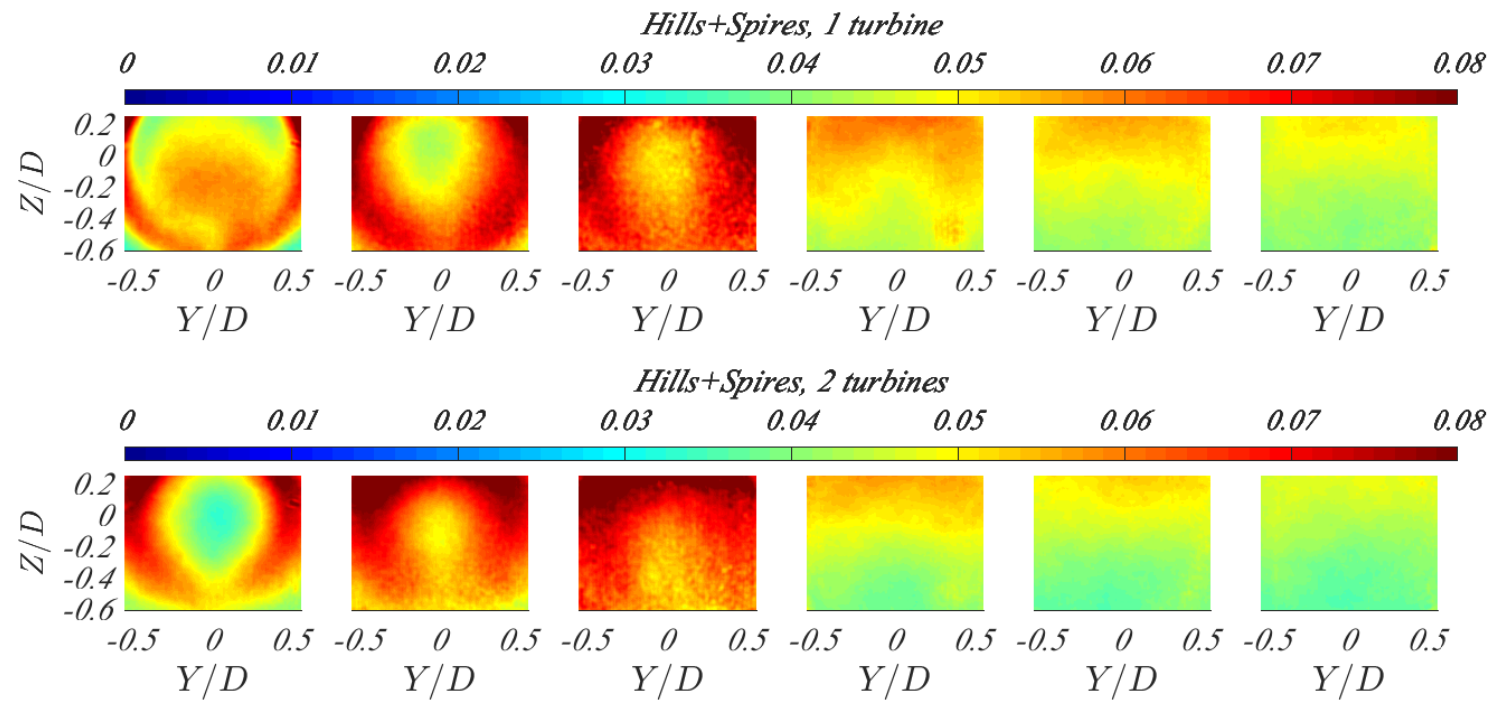

Figure 13. Normalised turbulent kinetic energy, $K / U_{\infty}^{2}$, in the wake of one turbine (top) and two turbines (bottom), with the hills+spires configuration. The planes (from left to right) are viewed at the streamwise locations (a-f) indicated in Figures $3 I-I I I$.
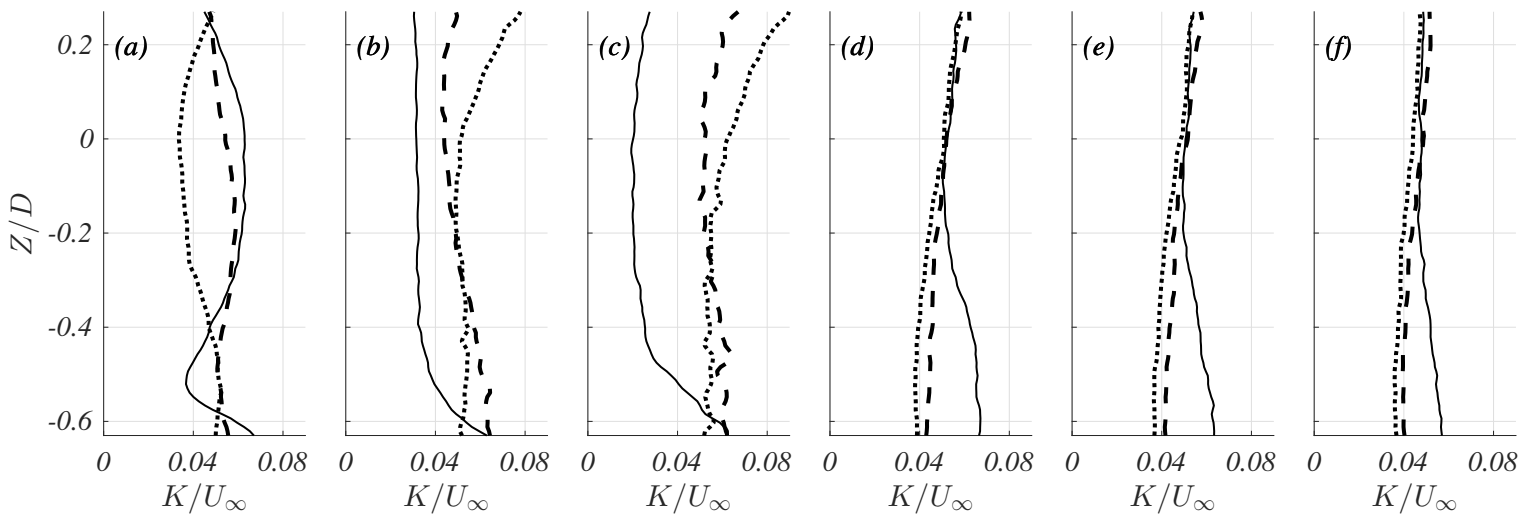

Figure 14. Normalised turbulent kinetic energy, $K / U_{\infty}^{2}$, along the rotor centreline with the hills+spires configuration, with one turbine (dashed lines) and two turbines (dotted lines). As a reference, the single-turbine case with hills is also included (solid lines). The streamwise locations where the profiles were measured are shown in Figures $3 I I-I I I$.

expected when considering the higher turbulence levels in the flow. Additionally, traces of similar flow patterns as in the homogeneous-inflow cases can be observed, where root- and tip-vortex regions are distinguishable in the first plane for the single-turbine wake, while only the tip-vortex region is visible to some extent when studying the multiple-turbine wake. The turbulent kinetic energy distribution along the rotor centreline is displayed in Figure 14 for these cases. Once more, the single-turbine case with hills is included as a reference. Clearly, the variations in the turbulent kinetic energy due to the presence of tip and root-vortices are much weaker in the single-turbine case with spires + grids, and even more so when two turbines are introduced. With the more turbulent inflow, the tip and root-vortex signatures in the turbulent kinetic energy field quickly vanishes with the downstream wake propagation, and they are hardly distinguishable beyond three diameters downstream of the rotor (Figure $14 \mathrm{~d}-\mathrm{f}$ ).

\subsection{Wake-centre movement under varying flow conditions}

To view how much the wake is deflected under the different flow configurations (in a time averaged sense), the vertical position of the maximum wake-velocity deficit, which in a sense can be viewed as the wake centre, is shown in Figure 

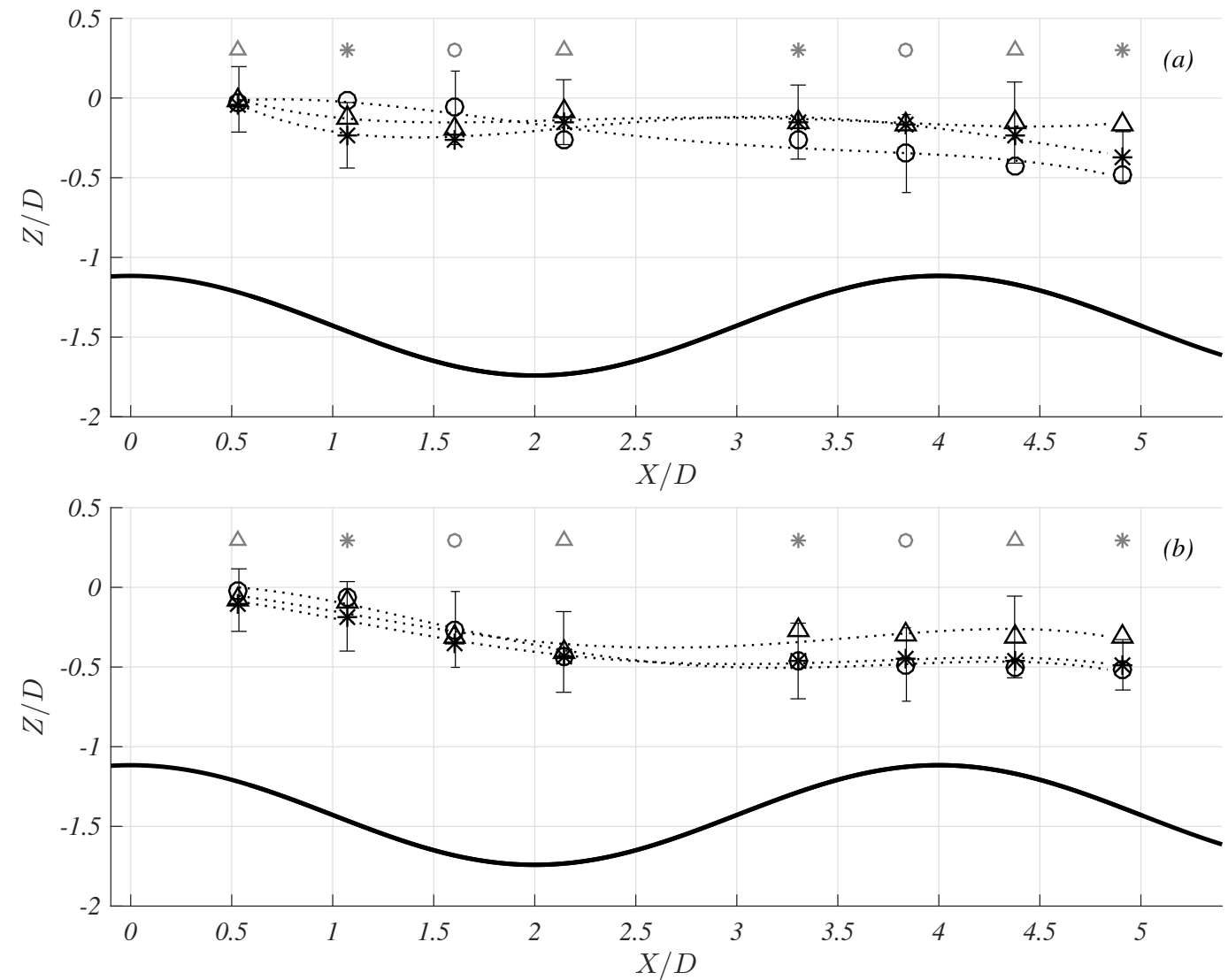

Figure 15. Vertical wake-centre movement with one turbine (a) and two turbines (b), for the different flow configurations plain $(\triangle)$, hills (*) and hills+spires (०). The hills are also indicated, assuming a reference frame origin at the hub height of the rearmost turbine. Curves following the data are plotted as visual aid. The error bars indicate the variability of the vertical wake-centre position in time (studying the time series of the flow configuration indicated by the overhead grey symbol).

15 Naturally, the wake will oscillate in time due to the wake-meandering phenomenon. This variability is indicated by the vertical bars in the figure and, as evident, the location of the maximum velocity deficit varies significantly over time. However, in a time-averaged sense, Figure 15 a shows that the wake-centre position is fairly constant for the singleturbine case with the plain configuration. With two turbines, a slight downward deflection can instead be seen for the plain configuration (Figure 15p), indicating that the tandem-turbine wake is influenced by the boundary-layer development over the flat surface. With hills the wake-centre position behind the single turbine (Figure 15 ) can be seen to roughly follow the hill curvature. Additionally, the downward wake deflection is stronger when introducing hills (compared to the plain configuration results), especially in the multiple-turbine case. With the hills +spires configuration, the centre position of the single-turbine wake is monotonically approaching the ground, and this occurs at a faster rate than for the homogeneousinflow configurations, which is probably associated to the higher velocity available at the top of the wake.

In the multiple-turbine wake, the centre position with hills+spires follows a similar pattern as the tandem-turbine case with hills. In both cases, the downward wake deflection is stronger than with the single-turbine configurations, although the wake deflection in the final planes could be underestimated due to the limited field of view. This suggests that the multipleturbine wakes are more influenced by the flow variations seen over the hills. In contrast, the horizontal variations of the time averaged wake-centre positions (not shown here) were considerably lower. It was evident that, while the horizontal variability of the instantaneous wake-centre positions was similar to the observed vertical variations (the standard deviation $\sigma_{\Delta Y} \approx \pm 0.25 D$ ), the time averaged values were close to zero for all flow cases. 


\section{SUMMARY}

An experimental analysis of the wake propagation behind one or two aligned wind turbines, over flat and hilly terrains, with homogeneous and sheared turbulent inflow conditions, has been performed. The hilly terrain consisted of a series of sinusoidal hills and the wind turbines were placed on the ridges of these. As expected, a more rapid wake diffusion was noted when introducing two turbines instead of one. Sheared turbulent inflow conditions further enhanced the wake diffusion, due to the increased turbulent mixing seen in the flow field. Another feature of the cases with large wake diffusion was the less distinct vortex regions, which could be seen when comparing the results for the multiple-turbine wakes with hills+spires (Figure 13, with the turbulent kinetic energy of the single-turbine wake with hills in Figure 9 As the wakes were diffused downstream of the turbines, their tip-vortex regions were broken down and became less distinct. At the same time, the wakes appeared to become more sensitive to the vertical flow perturbations introduced by the hills, resulting in larger downward wake deflections.

Over flat terrain, the vertical position of the single-turbine wake was fairly constant, while the tandem-turbine wake tended towards the ground as it propagated downstream. When introducing the hilly terrain, the wake centre was instead seen to roughly follow the hill curvatures. An enhanced wake deflection could furthermore be seen with hills, which was stronger for the multiple-turbine wake than for the single-turbine wake.

The observations made above can also be viewed in a wider perspective. As seen in the single-turbine cases with an homogeneous inflow, flow situations with low ambient turbulence levels (with wakes featuring distinct vortex regions) seem to be less affected by external flow perturbations and their wake diffusion is slower. This can be compared to fullscale situations where wind-turbine wakes are seen to propagate over longer distances under low-turbulence atmospheric conditions. It also indicates that external vertical velocity variations induced by hilly terrains are important to consider in highly-turbulent turbulent flow situations, which generally is the case of wind turbines operating in field conditions. The larger wake diffusion seen with hills shows that complex terrains may be beneficial for wind farms where wake-interaction effects significantly reduce the power output.

As seen in the results of the present study, more diffused wakes are more susceptible to deflect in response to hillinduced vertical velocity variations. Therefore, when looking at wake models applied to simulations over hilly terrain, accurate turbulence modelling is crucial to properly capture the wake development. Although full-scale wind turbines are significantly larger than the wind-turbine models used in this study, the differences in the flow fields observed under the various measurement conditions studied here are qualitatively relevant and should be considered in full-scale situations as well.

\section{ACKNOWLEDGEMENT}

The work presented in the present paper has been funded by The Swedish Research Council (VR) under a framework grant for strategic energy research. The authors wish to acknowledge Jan-Åke Dahlberg at Vattenfall Vindkraft AB for lending equipment to the measurement campaign and for manufacturing the wind-turbine rotors. Jonas Vikström and Rune Lindfors at KTH are acknowledged for manufacturing items used in the wind-tunnel setup. Dr. Bengt Fallenius at KTH is acknowledged for his support with setting up the measurement equipments while Prof. P. Henrik Alfredsson is acknowledged for giving useful feedback during the work. A. Segalini is supported by the Swedish strategic research program STandUP for Wind.

\section{REFERENCES}

1. S. Emeis, Wind Energy Meteorology, ch. 4, Winds in complex terrain. Springer-Verlag Berlin Heidelberg, 2013. 
2. C. Schulz, L. Klein, P. Weihing, T. Lutz, and E. Krämer, "CFD studies on wind turbines in complex terrain under atmospheric inflow conditions," Journal of Physics: Conference Series, vol. 524, no. 1, p. 012134, 2014.

3. C. G. Helmis, K. H. Papadopoulos, D. N. Asimakopoulos, P. G. Papageorgas, and A. T. Soilemes, "An experimental study of the near-wake structure of a wind turbine operating over complex terrain," Solar Energy, vol. 54, no. 6, pp. 413-428, 1995.

4. D. R. Webster, D. B. DeGraaff, and J. K. Eaton, "Turbulence characteristics of a boundary layer over a twodimensional bump," Journal of Fluid Mechanics, vol. 320, pp. 53-69, August 1996.

5. S. P. S. Arya, M. E. Capuano, and L. C. Fagen, "Some fluid modeling studies of flow and dispersion over twodimensional low hills," Atmospheric Environment, vol. 21, no. 4, pp. 753-764, 1987.

6. C. A. Miller and A. G. Davenport, "Guidelines for the calculation of wind speed-ups in complex terrain," Journal of Wind Engineering and Industrial Aerodynamics, vol. 74, pp. 189-197, 1998.

7. H. G. Kim, C. M. Lee, H. C. Lim, and N. H. Kyong, "An experimental and numerical study on the flow over twodimensional hills," Journal of Wind Engineering and Industrial Aerodynamics, vol. 66, no. 1, pp. 17-33, 1997.

8. K. Røkenes and P. Å. Krogstad, "Wind tunnel simulation of terrain effects on wind farm siting," Wind Energy, vol. 12, pp. 391-410, 2009.

9. P. S. Jackson and J. C. R. Hunt, “Turbulent wind flow over a low hill," Quarterly Journal of the Royal Meteorological Society, vol. 101, pp. 929-955, 1975.

10. J. J. Finnigan, "Air flow over complex terrain," in Flow and transport in the natural environment: advances and applications (W. L. Steffen and O. T. Denmead, eds.), pp. 183-229, Springer, Berlin, Heidelberg, 1988.

11. A. D. Ferreira, M. C. G. Silva, D. X. Viegas, and A. G. Lopes, "Wind tunnel simulation of the flow around twodimensional hills," Journal of Wind Engineering and Industrial Aerodynamics, vol. 38, pp. 109-122, 1991.

12. P. Carpenter and N. Locke, "Investigation of wind speeds over multiple two-dimensional hills," Journal of Wind Engineering and Industrial Aerodynamics, vol. 83, no. 1, pp. 109-120, 1999.

13. K. B. Howard, L. P. Chamorro, and M. Guala, "A comparative analysis on the response of a wind-turbine model to atmospheric and terrain effects," Boundary-Layer Meteorology, vol. 158, pp. 229-255, 2016.

14. X. Yang, K. B. Howard, M. Guala, and F. Sotiropoulos, "Effects of a three-dimensional hill on the wake characteristics of a model wind turbine," Physics of Fluids, vol. 27, p. 025103, 2015.

15. N. Troldborg, J. N. Sørensen, and R. Mikkelsen, "Actuator line simulation of wake of wind turbine operating in turbulent inflow,” Journal of Physics: Conference Series, vol. 75, no. 1, p. 012063, 2007.

16. P. Mycek, B. Gaurier, G. Germain, G. Pinon, and E. Rivoalen, "Experimental study of the turbulence intensity effects on marine current turbines behaviour. Part I: One single turbine,” Renewable Energy, vol. 66, pp. 729-746, June 2014.

17. P. Mycek, B. Gaurier, G. Germain, G. Pinon, and E. Rivoalen, "Experimental study of the turbulence intensity effects on marine current turbines behaviour. Part II: Two interacting turbines," Renewable Energy, vol. 68, pp. 876-892, August 2014.

18. M. S. Adaramola and P. A. Krogstad, "Experimental investigation of wake effects on wind turbine performance," Renewable Energy, vol. 36, pp. 2078-2086, 2011.

19. F. Castellani, D. Astolfi, M. Burlando, and L. Terzi, "Numerical modelling for wind farm operational assessment in complex terrain," Journal of Wind Energy and Industrial Aerodynamics, vol. 147, pp. 320-329, December 2015.

20. F. Castellani, D. Astolfi, M. Mana, E. Piccioni, M. Becchetti, and L. Terzi, "Investigation of terrain and wake effects on the performance of wind farms in complex terrian using numerical and experimental data," Wind Energy, vol. 20, no. 7, pp. 1277-1289, 2017.

21. W. Tian, A. Ozbay, W. Yuan, P. Sarakar, and H. Hu, "An experimental study on the performances of wind turbines over complex terrain," in 51st AIAA Aerospace Sciences Meeting including the New Horizons Forum and Aerospace Exposition, (Grapevine, Texas), AIAA 2013-0612, January 2013.

22. W. Tian, A. Ozbay, W. Yuan, and H. Hu, "An experimental investigation on the performance of wind turbines sited over a hilly terrain," in 31st AIAA Applied Aerodynamics Conference, AIAA 2013-2804, 2013. 
23. J. L. Walmsley, I. Troen, L. D. P., and P. J. Mason, "Surface-layer flow in complex terrain: comparison of models and full-scale observations," Boundary-Layer Meteorology, vol. 52, no. 3, pp. 259-281, 1990.

24. D. E. Neff and R. N. Meroney, "Wind-tunnel modelling of hill and vegetation influence on wind power availability," Journal of Wind Energy and Industrial Aerodynamics, vol. 74, pp. 335-343, 1998.

25. R. B. Stull, An Introduction to Boundary Layer Meteorology. Kluwer Academic Publishers, 1988.

26. J. Cheng, D. Lubitz, and B. R. White, "Wind-tunnel prediction of wind power production in complex terrain," in 42nd AIAA Aerospace Sciences Meeting and Exhibit, AIAA 2004-1360, 2004.

27. N. J. Vermeer, J. N. Sørensen, and A. Crespo, "Wind turbine wake aerodynamics," Progress in Aerospace Sciences, vol. 39, pp. 467-510, 2003.

28. B. Sanderse, S. P. van der Pijl, and B. Koren, "Review of computational fluid dynamics for wind turbine wake aerodynamics," Wind Energy, vol. 14, pp. 799-819, 2011.

29. L. P. Chamorro, R. E. A. Arndt, and F. Sotiropoulos, "Reynolds number dependence of turbulence statistics in the wake of wind turbines," Wind Energy, vol. 15, pp. 733-742, 2011.

30. L. P. Chamorro and F. Porté-Agel, "A wind-tunnel invesitgation of wind-turbine wakes: boundary-layer turbulence effects,” Boundary-Layer Meteorology, vol. 132, pp. 129-149, 2009.

31. I. Dobrev and F. Massouh, "Development of near wake of a HAWT depending on hub height," in Proceedings of the 5th International Conference on Jets, Wakes and Separated Flows (ICJWSF2015) (A. Segalini, ed.), Springer Proceedings in Physics 185, 2016.

32. B. Lindgren and A. V. Johansson, "Design and evaluation of a low-speed wind-tunnel with expanding corners," TRITA-MEK Technical report 2002:14, KTH Royal Institute of Technology, 2002.

33. H. P. A. H. Irwin, "The design of spires for wind simulation," Journal of Wind Engineering and Industrial Aerodynamics, vol. 7, pp. 361-366, 1981.

34. A. Hyvärinen and A. Segalini, "Effects from complex terrain on wind-turbine performance," Journal of Energy Resources Technology, vol. 139, p. 051205, September 2017.

35. J. J. Nelka, "Evaluation of a rotating disk apparatus: drag of a disk rotating in a viscous fluid," Tech. Rep. 3851, David W Taylor Naval Ship Reasearch and Development Center, Bethesda MD Ship Performance Dept., 1973.

36. S. Imayama, P. H. Alfredsson, and R. J. Lingwood, "A new way to describe the transition characteristics of a rotatingdisk boundary-layer flow," Physics of Fluids, vol. 24, p. 031701, 2012.

37. M. Raffel, C. E. Willert, S. Wereley, and J. Kompenhans, Particle Image Velocimetry: A practical guide. Springer, 2 ed., 2007. 